\title{
WAVES AND OSCILLATIONS IN THE CHROMOSPHERE IN ACTIVE AND QUIET REGIONS
}

\author{
R. G. GIOVANELLI \\ CSIRO Division of Physics, National Standards Laboratory, Sydney, Australia 2008
}

\begin{abstract}
Oscillations and waves have been observed in $\mathrm{H} \alpha$ in sunspot umbras and penumbras. They appear to be basically of the Alfvén type, but do not carry away sufficient energy to account for the sunspot energy deficit.

There is a pronounced difference in the velocity structures of supergranule centres and boundaries. In the former it is oscillatory, of shorter period but bigger amplitude than in the photosphere. In the network there are oscillations of the fibrils but, on the whole, little evidence of wave motion along their lengths except perhaps near their diffuse ends.

In plages, there are velocity oscillations which at times may be phase coherent over extensive regions up to $10^{5} \mathrm{~km}$ apart; there are suggestions of a connection between these times and the occurrence of flares. There is also a report of a velocity pulse travelling along an active-region fibril with a velocity of propagation of $150 \mathrm{~km} \mathrm{~s}^{-1}$.
\end{abstract}

\section{Introduction}

For several years following Leighton et al. (1962) discovery of photospheric oscillations, their behaviour was studied almost exclusively via time variations in spectra. But in the past four years there has been a growing tendency to use spectroheliographs and filters, as a result of which we are getting more-detailed two-dimensional information. The most spectacular result has been the discovery of waves propagating outwards over sunspot penumbras. We trace below the discovery of these waves and the associated umbral oscillations, and review present knowledge of oscillations and waves in quiet and active parts of the chromosphere.

\section{Sunspots}

Sunspot umbras exhibit vertical velocity oscillations at low levels. Beckers and Schultz (1972), using Fe 5434 which has zero Zeeman splitting, found a peak-to-peak amplitude of $1 \mathrm{~km} \mathrm{~s}^{-1}$ and a period of $178 \mathrm{~s}$ in one umbral centre, and a longer period of $255 \mathrm{~s}$ at the umbral-penumbral boundary; in four other umbras there were no apparent motions. Bhatnagar et al. (1972), using unidentified lines formed only in sunspots and also with zero Zeeman splittings, found peak-to-peak amplitudes of $0.5 \mathrm{~km} \mathrm{~s}^{-1}$ and periods of $448 \mathrm{~s}(6021 \AA)$ and around $310 \mathrm{~s}(6252,6910 \AA)$ in one sunspot. In Fe II 4924, Sheeley and Bhatnagar (1971) had earlier found no umbral or penumbral oscillations, perhaps because of inadequate sensitivity, while Beckers and Schultz (1972) found no intensity oscillations in the continuum. There are therefore clear differences in the strengths of oscillations observed in different umbras and at different heights though, as Bhatnagar et al. (1972) indicate, the amplitudes are less than in the normal photosphere. We should reserve judgment on whether the reported differences in period in 
different lines are significant until observations have been made simultaneously at the one position in these lines.

The first evidence of sunspot oscillations in a chromospheric line was presented by Beckers and Tallant (1969), who described umbral 'flashes' in the K line, of diameter up to $2000 \mathrm{~km}$, tending to repeat at intervals up to $145 \mathrm{~s}$. Individual flashes lasted about $50 \mathrm{~s}$, with a faster rise than decay. There were associated transverse velocities of some $40 \mathrm{~km} \mathrm{~s}^{-1}$ towards the penumbra, though these were thought unlikely to be due to material motions. Doppler shifts showed the vertical velocities to be mainly upwards at 6-7 $\mathrm{km} \mathrm{s}^{-1}$, though there was a brief, rapid change to a downflow just before the next flash. Flashes in the IR $\mathrm{Ca}^{+}$line occurred virtually simultaneously (Beckers and Schultz 1972), but while some umbral flashes occurred in $\mathrm{H} \alpha$, these were much less frequent. No connection was found between flashes and lower-level umbral velocities in $\mathrm{Fe} 5434$.

While the nature of umbral flashes remains vague, interest has swung recently to waves and oscillations observable in $\mathrm{H} \alpha$. At Big Bear, Stein and Zirin(1972, and private communication) have observed sunspots through an $\mathrm{H} \alpha$ filter, finding waves propagating outwards over the penumbra at velocities typically $8-12 \mathrm{~km} \mathrm{~s}^{-1}$. They reported these as usually seen best at line centre, but occasionally in the wing out to $0.7 \AA$. They occur in almost every sizable spot with a stable regular penumbra, but rarely in active spots with complex structure. In almost all cases the period lay between 4 and 5 min, the shortest observed being $150 \mathrm{~s}$ in a spot of particularly strong magnetic field. They inclined to the view that these represented sound waves.

Independently, at Culgoora, Giovanelli (1972) studied sunspots through an $\mathrm{H} \alpha$ filter, using a polarizing beam-splitter to secure photographs at effectively $0.25 \AA$ from line centre simultaneously in opposite wings. Subtraction of one from the other yields the line-of-sight velocity distribution. The resulting films of sunspots near disk centre also showed waves propagating outwards over the penumbra (Figure 1). The first sunspot measured at Culgoora had periods of the order of $210 \mathrm{~s}$ and velocities of $15-25 \mathrm{~km} \mathrm{~s}^{-1}$. Measurements on this spot over a longer time interval and on other spots favour $15 \mathrm{~km} \mathrm{~s}^{-1}$ as more typical (Table I), though wavelength (which is not easy to measure) and period vary appreciably. The peak line-of-sight velocity, $v$, measurable only with difficulty, is of the order of $1 \mathrm{~km} \mathrm{~s}^{-1}$. The waves observed at Big Bear and Culgoora are undoubtedly the one phenomenon though the measured properties differ somewhat; some at least of the differences are due to real differences between one spot and another.

The wave mode can be studied using sunspots at different positions on the disk. Longitudinal modes, as in acoustic waves, would give Doppler shifts for spots near the limb when the waves propagate along the disk radius vector. Transverse waves with horizontal displacements can be studied in spots near the limb when the waves propagate at right angles to the line of sight. We have looked for modes of these types but failed to find any trace; at most, they are below our resolution limits.

Searches have also been made for waves with an $0.7 \AA$ filter centred on $\mathrm{H} \alpha$; with this pass band, pure Doppler shifts without an associated change in depth of the line 

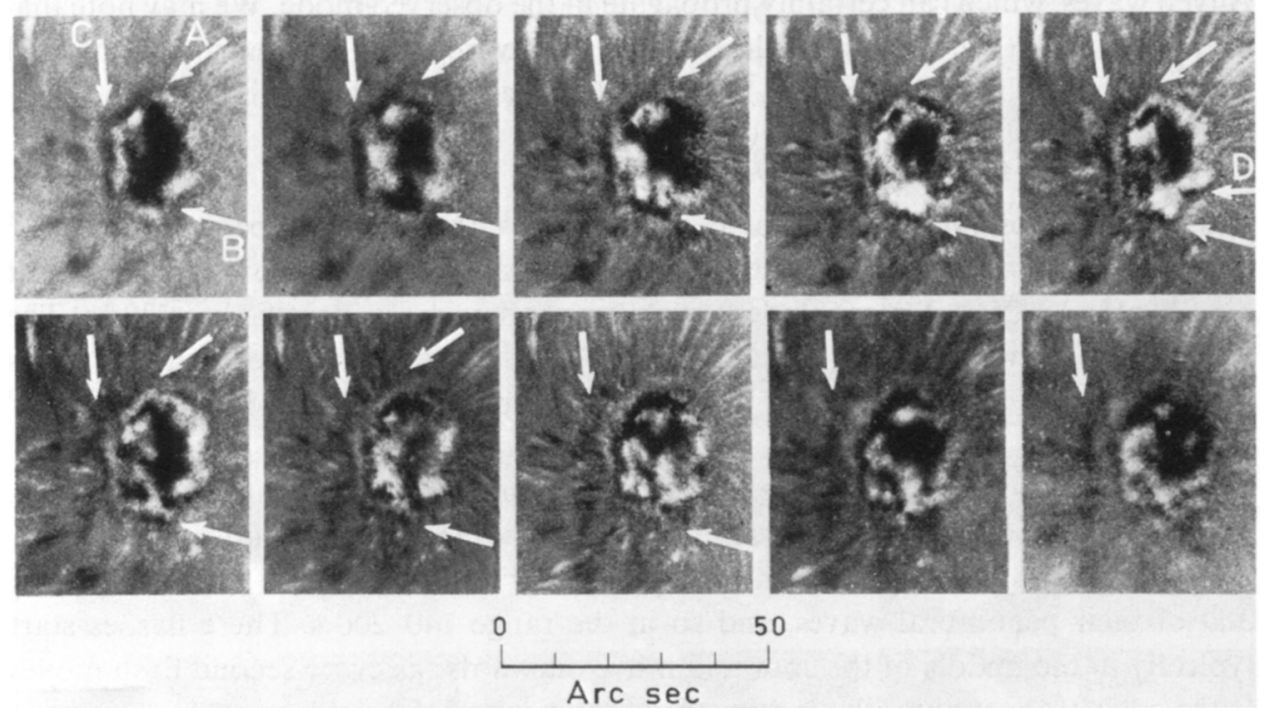

50

Arc sec

Fig. 1. H $\alpha$ velocity distributions across a sunspot and surrounding regions at $30-\mathrm{s}$ intervals, obtained by subtracting simultaneous photographs obtained in opposite wings at $\pm 0.25 \AA$ from line centre. Dark indicates downward velocities, bright upward. $A, B$ and $C$, and the corresponding arrows on the various frames, point to and lie in the same line as progressive positions of wave fronts moving outwards from the umbra across the penumbra.

should produce no change in the penumbral intensity. No intensity waves have been detected, in conflict with Stein and Zirin's result. Nor have such waves been detected in the continuum or in velocities in $\mathrm{Fe}$ 6569. We have searched for the latter using the $1 / 8 \AA$ filter and a polarizing beamsplitter giving images at $\pm 1 / 16 \AA$ from line centre simultaneously, but whether velocity waves are absent in this line or the observing technique has been inadequate is just not known. Further observations are needed.

The nature of the wave follows from these results. The velocity observed by Giovanelli exceeds the sound velocity considerably, though Stein and Zirin's velocity is close to that of sound. Preferring my own results, I infer that they cannot be basically acoustic waves - and in any case the observed displacement is transverse, not longitudinal as in acoustic waves. Gravity waves propagate much more slowly. There remain only

TABLE I

Properties of penumbral waves (author's observations)

\begin{tabular}{llll}
\hline Sunspot date & Period $(\mathrm{s})$ & Wavelength $\left(10^{3} \mathrm{~km}\right)$ & $V\left(\mathrm{~km} \mathrm{~s}^{-1}\right)$ \\
\hline $71-$ VII-4 & 240 & 3.35 & 14 \\
$71-$ XI-3 & 210 & $3.1-5.2$ & $15-25$ \\
& 200 & 2.35 & 12 \\
$72-$ III-10 & 230 & 2.75 & 12 \\
$72-$ IV-21 & 180 & 3.8 & 21 \\
\hline
\end{tabular}


Alfvén waves, which can certainly propagate in the observed mode. We may note that in the penumbra the magnetic field is radially outwards, and inclined at only a small angle to the horizontal; typically, $H \approx 1200 \mathrm{G}$. The Alfvén velocity is

$$
V_{\mathrm{A}}=H /(4 \pi \varrho)^{1 / 2}
$$

and with the observed velocity of $V_{\mathrm{A}}=15 \mathrm{~km} \mathrm{~s}^{-1}$, the density $\varrho$ becomes $5 \times 10^{-8}$ $\mathrm{gm} \mathrm{cm}^{-3}$. This fits about as closely as we could guess to the penumbral density at $\tau \approx 1$ for $\mathrm{H} \alpha \pm 0.25 \AA$. We conclude that the waves have been identified as Alfvén. The total flux in the wave, $\frac{1}{2} \varrho v^{2} V_{\mathrm{A}}$ per unit area normal to the direction of propagation, is $4 \times 10^{8} \mathrm{erg} \mathrm{cm}^{-2} \mathrm{~s}^{-1}$, much less than the photospheric radiation flux of $6.4 \times 10^{10}$ erg $\mathrm{cm}^{-2} \mathrm{~s}^{-1}$.

Is the velocity uniform or accelerated? One cannot give a definite answer as yet, though at times one has the impression of a positive acceleration outwards.

In the umbra, Stein and Zirin have reported $H \alpha$ flashes of period invariably $\frac{1}{2}$ to $\frac{2}{3}$ that of their penumbral waves, and so in the range 140-200 s. These flashes start typically in the middle of the spot and move outwards; as every second flash moves to the edge of the umbra, the inner edge of the penumbra brightens and a new wave begins. It is by no means established whether these are related to the umbral flashes in $\mathrm{K}$. However it is almost certain that they are related to umbral oscillations observed in $\mathrm{H} \alpha$ by Giovanelli (1972). Using the beamsplitter technique described above, he has found two interesting properties of all umbras studied:

(i) Near disk centre there is a quasi-oscillatory velocity distribution (Figure 2) whose structural elements have a size of the order of $3^{\prime \prime}$, a period typically $165 \mathrm{~s}$, and velocity amplitude of the order of $3 \mathrm{~km} \mathrm{~s}^{-1}$. In a 30-min set of observations, oscillations of individual points were followed for almost the whole sequence, but the pattern as a whole broke down after 1 or 2 periods since the individual points have differing periods. In one umbra, periods of 137 and $185 \mathrm{~s}$ were recorded at different points.

(ii) There is a fascinating behaviour of the locus of points of the same phase, i.e. the wavefront. This undergoes continual change. New wavefronts often develop near the centre of the umbra, though they may be eccentric or appear at the edge of the umbra and move across to the opposite side. They sometimes swirl around the umbra, and the sense of swirling can change rapidly from clockwise to anticlockwise or vice versa, demonstrating that at a given time the period varies across the umbra, and changes with time at a given point. On the whole, the wavefronts progress outwards towards the penumbra, but their behaviour is far more complicated than a mere symmetrical expansion from the centre outwards.

Giovanelli, also, finds that there is a close relation between umbral oscillations and penumbral waves; the latter run smoothly out of the umbral waves. Both form part of the one wave system (Giovanelli's measured differences in period are almost certainly due to sampling, and of no physical significance apart from forming additional examples of the variability of period from point to point and in time). Specifically, this demonstrates that there is not merely energy storage in the oscillations, but a flux of energy out of the umbra and across the penumbra. Simultaneous observations are 


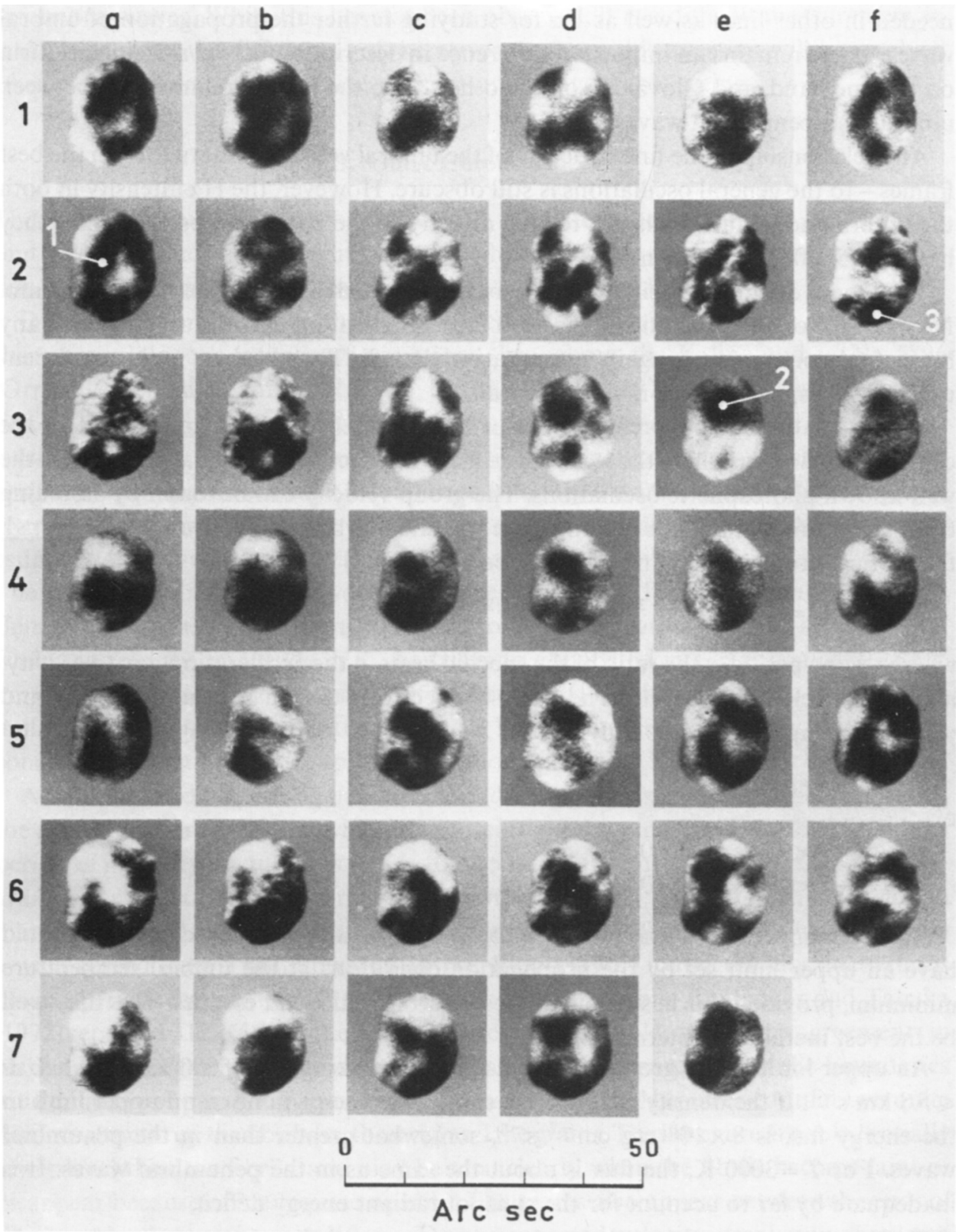

Fig. 2. Array of $\mathrm{H} \alpha$ velocities in an umbra at 30-s intervals. The sharp boundary is due to instrumental saturation. Point No. 1 is oscillatory with maximum downward velocities on frames $1 \mathrm{~b}, 2 \mathrm{a}, 2 \mathrm{e}, 3 \mathrm{e}, 4 \mathrm{~b}, 5 \mathrm{a}$, $5 \mathrm{f}, 6 \mathrm{~d}$. Two other oscillatory points are 2 (frames $1 \mathrm{a}, 2 \mathrm{a}, 2 \mathrm{f}, 3 \mathrm{e}, 4 \mathrm{~d}, 5 \mathrm{~d}, 6 \mathrm{e}$ ) and 3

(frames 1a, 2a, 2f, 3f, 4f, 6a, 7b). 
needed in other lines as well as $\mathrm{H} \alpha$ for studying further the propagation of umbral waves. There remains an important difference in description between Stein and Zirin on the one hand, and Giovanelli on the other, as to the precise relationship between umbral and penumbral waves.

Any relationship of the fine structure of the umbral velocity pattern - 1 " on the best frames - to the general oscillations is still obscure. However, the $\mathrm{H} \alpha$ intensity in both the umbra and penumbra is not related directly to the pattern of oscillations - they have quite differing structures.

The modes of umbral oscillation have been examined in large umbras near the limb. No evidence of any oscillations has been found, indicating that the amplitude of any horizontal component is again comparatively small. Thus both in umbra and penumbra the displacement is mainly vertical.

The oscillations are therefore more-or-less parallel to the magnetic field in the centre of the umbra, where the wave mode must be effectively identical with that in the well-known photospheric oscillations. The group velocity can be found by assuming them to be adiabatic. For a region where the undisturbed temperature is isothermal, the vertical group velocity may be written

$$
U=C_{\mathrm{s}}\left(1-\frac{1}{16} \pi^{-2 t 2} \gamma^{2} g^{2} C_{\mathrm{s}}^{-2}\right)^{1 / 2},
$$

where $t$ is the period, $\gamma$ the ratio of the specific heats, $g$ the acceleration due to gravity, and $C_{\mathrm{s}}$ the velocity of sound. The latter is effectively that in neutral hydrogen, and $\gamma=5 / 3$. Propagation occurs only when

or

$$
t \gamma g<4 \pi C_{\mathrm{s}},
$$

$$
\begin{array}{r}
t<228 \mathrm{~s} \text { at } 5000 \mathrm{~K}, \\
<177 \mathrm{~s} \text { at } 3000 \mathrm{~K} .
\end{array}
$$

Umbral temperatures may be close to $3000 \mathrm{~K}$, and the observed periods should have an upper limit set by the propagation conditions at the umbral temperature minimum, provided this lies below the region from which $\mathrm{H} \alpha$ escapes. This may well be the best method of determining $T_{\min }$.

An upper limit to the group velocity is given by taking $T$ as $5000 \mathrm{~K}$, so that $U$ is $\leqslant 5.8 \mathrm{~km} \mathrm{~s}^{-1}$. If the density is about the same as in the penumbra, an upper limit to the energy flux is $8 \times 10^{8} \mathrm{erg} \mathrm{cm}^{-2} \mathrm{~s}^{-1}$, somewhat greater than in the penumbral waves. For $T=3000 \mathrm{~K}$, the flux is about the same as in the penumbral waves. It is inadequate by far to account for the sunspot radiant energy deficit.

The penumbral waves are obviously due to cross-coupling between the effectively vertical gas motions in the umbra and the sunspot magnetic field, which becomes more and more inclined from the vertical on going towards and into the penumbra. The force tubes are waved up and down, generating Alfvén waves with a predominantly vertical displacement.

It is not yet known why the waves disappear at the outer penumbral boundary. The decreasing density outwards should result in an outward acceleration of the 
penumbral waves. While this has not been observed, it is a difficult measurement to make because of the diffuseness of the waves. Perhaps this question will be resolved when we can observe a very large sunspot under favourable seeing.

\section{Quiet Chromosphere}

There are obvious differences between the oscillatory behaviours of supergranule centres and boundaries. Most investigators have used spectrograms, the test of location on supergranule structures being the intensity in the $\mathrm{K}$ or $\mathrm{H} \alpha$ line.

Over supergranule centres, the period of velocity fluctuations decreases with height; there are analogous intensity fluctuations throughout the $\mathrm{K}$ line and in $\mathrm{Na} 5896$ (Orrall, 1966; Tanenbaum et al., 1969; Liu and Sheeley, 1971). At the height of formation of $\mathrm{K}_{1}$ and $\mathrm{K}_{2}$, the intensity period is about $250 \mathrm{~s}$ (Jensen and Orrall 1963). At the level of $K_{1}$, intensity maxima lead upwards velocity maxima in photospheric lines by about $90^{\circ}$ in phase, indicating adiabatic conditions and standing, rather than running, sound waves. Tanenbaum et al. (1969) found similar conditions for $\mathrm{Na} 5896$; they also estimated the coherence length (the size of the region of uniform phase) to be $3000 \mathrm{~km}$. The period is significantly lower in the cores of $\mathrm{H} \alpha$ and $\mathrm{K}_{3}$, in the range 150-210 s (Jensen and Orrall, 1963; Orrall, 1966; Elliott, 1969; Bhatnagar and Tanaka, 1972), while the velocity amplitude in $\mathrm{K}_{3}$ has risen to about $1.6 \mathrm{~km} \mathrm{~s}^{-1}$ as against $0.25-0.5$ $\mathrm{km} \mathrm{s}^{-1}$ in the photosphere (Orrall, 1966). Deubner (1971) has reported a more-or-less uniform distribution of amplitudes up to $3 \mathrm{~km} \mathrm{~s}^{-1}$ in $\mathrm{H} \alpha$, so that $\mathrm{H} \alpha$ and $\mathrm{K}_{3}$ give concordant mean velocity amplitudes of about $1.5 \mathrm{~km} \mathrm{~s}^{-1}$.

A wider spread of periods has been found at supergranule boundaries, for which the results appear to be somewhat more discordant. Liu and Sheeley (1971) found the period of intensity fluctuations in $\mathrm{K}_{2 v}$ to be $5 \mathrm{~min}$, substantially longer than in supergranule centres, and they reported bigger amplitudes also. In $\mathrm{K}_{3}$, Orrall (1966) found many periods of velocity fluctuations grouping around $180 \mathrm{~s}$, and there was also quite a wide spread from 5 min upwards. In $\mathrm{H} \alpha$, Elliott (1969) found velocity fluctuations having periods around 150-210, 287 and about $900 \mathrm{~s}$, while Bhatnagar and Tanaka (1972) reported $312 \pm 56 \mathrm{~s}$ in the bright centres of rosettes. However, the agreements in respect of supergranule centres and mild disagreements in respect of the boundaries hide an array of puzzles. For example, in $\mathrm{H} \alpha$, supergranule centres contain two quite distinct structures, the fibrils (or threads) and the grains. The latter are at a substantially lower level than the fibrils, and may be seen clearly when the fibrils become faint or disappear because of inadequate opacity. The grains also appear between the ends of fibrils under favourable conditions. Do the reported velocity oscillations apply to both these structures, or to one only? Supergranule boundaries too contain quite a pronounced structure, and one wonders what differences there may be from point to point.

Velocity films in $\mathrm{H} \alpha$ hold out the hope of clarifying such issues. Films of this type obtained at Culgoora - similar to those of Ramsay at Lockheed - show a vigorous state of oscillation, with quite distinct patterns in different regions. 
(a) The Grains. In the central portions of supergranule centres - i.e. in those regions where we can see the grains clearly - the regions of oscillation have no particular shape, and appear to be distributed more or less at random. I have sought evidence of any propagation of these oscillations across the surface of the sun but have found none. It would be interesting to compare the patterns of oscillation in $H \alpha$ and $K_{3}$, and in $\mathrm{H} \alpha$ and lines from underlying regions, using simultaneous filtergrams or spectroheliograms obtained at intervals of at most $15 \mathrm{~s}$ and preferably much less.

(b) The Fibrils. Where the fibrils are well developed, the system of motion is quite different and is still inadequately studied. However the regions of uniform phase appear to be elongated and aligned parallel to the pattern of the fibrils, and this is particularly noticeable where the fibril alignment varies rapidly from place to place here the individual elongated oscillating regions copy faithfully the varying fibril directions.

It is not eacy to locate the place where pattern (a) gives way to pattern(b). The former seems to extend somewhat into the fibril zone, but whether this means that the diffuse ends of the fibrils partake of the oscillations of the grainy region or that we are able to see the underlying region through or between fibrils is uncertain.

On the whole, the oscillations in the regions of the fibrils do not exhibit obvious wave motions along their lengths. However, we occasionally find places where there seem to be motions along the fibrils and these are generally located near the diffuse ends. It is probable that they represent Alfvén waves, but better observations are needed.

At supergranule boundaries, velocity fluctuations are of much reduced amplitude at the level corresponding to $\mathrm{H} \alpha \pm 0.25 \AA$, quite the opposite of what Liu and Sheeley found for intensity fluctuations in $\mathrm{K}_{2 v}$. There is little correlation between intensity in $\mathrm{H} \alpha$ and direction of motion at this wavelength, a result in good agreement with Grossman-Doerth and von Uexküll's $(1971,1973)$ ffndings. Velocity films have not yielded obvious patterns of the behaviour with time; there is need for a much better study than any reported so far.

\section{Active Regions}

Fibrils near sunspots are almost certainly of the same basic structure as those in the quiet chromosphere. Bhatnagar and Tanaka (1972) have examined one region where the fibrils merged together to produce an almost structureless pattern, but failed to find any oscillations in $\mathrm{H} \alpha-0.5 \AA$. Giovanelli has recently been examining fibrils in active regions, finding that on the whole these too exhibit very few velocity fluctuations. However, these are occasional events which almost certainly represent Alfvén waves (Figure 3), a velocity pulse running along a fibril at a propagation speed of the order of $150 \mathrm{~km} \mathrm{~s}^{-1}$. Much more study needs to be given to these events, including their connection, if any with changes in the appearance of fibrils at line centre.

Bhatnagar and Tanaka (1972) have studied intensity oscillations in plages at $\mathrm{H} \alpha$ $-0.5 \AA$, finding periods of $282 \pm 49 \mathrm{~s}$. Subsequently Tanaka (private communication) was able to demonstrate that these were oscillations mainly of velocities. He found that 


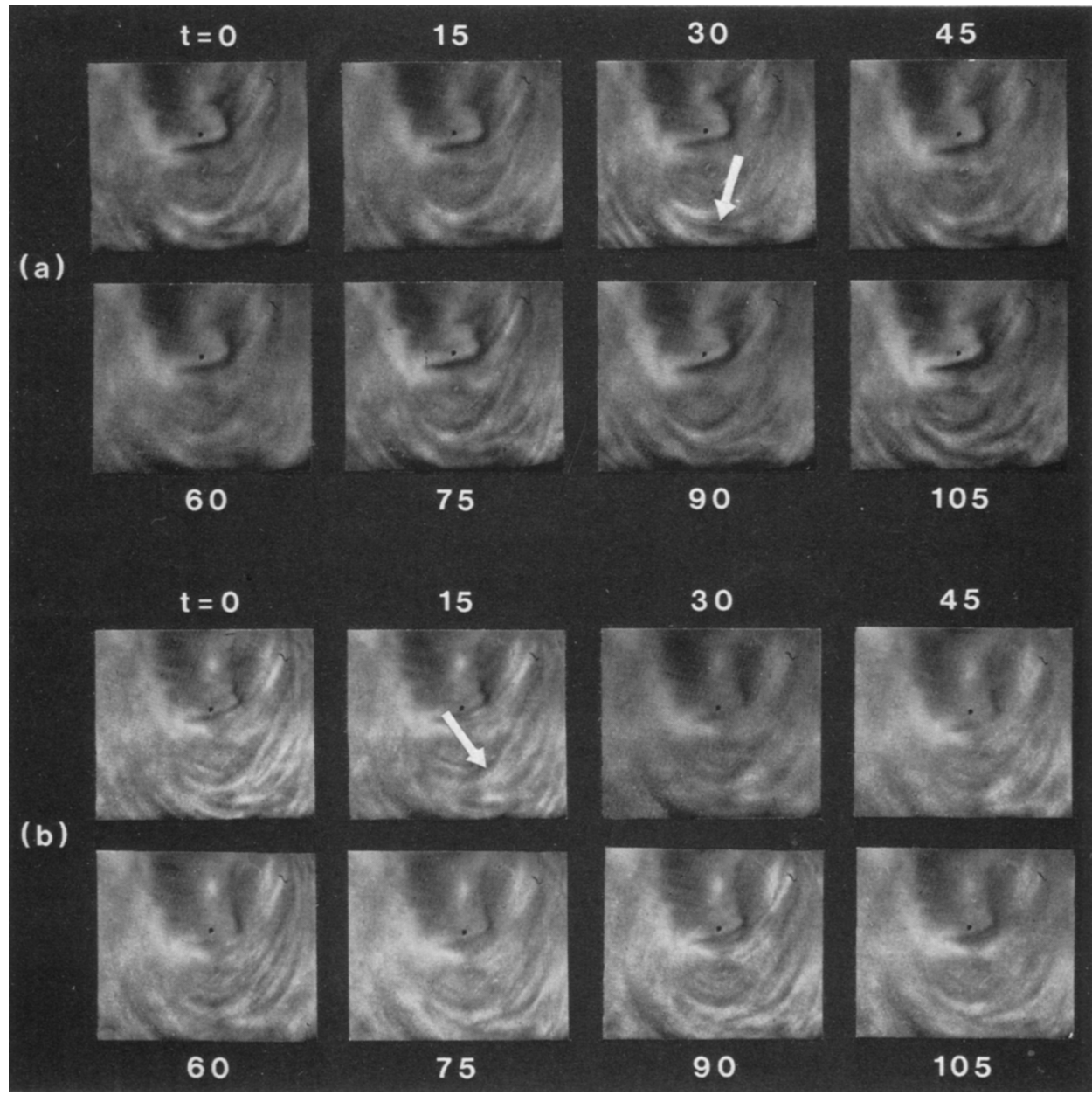

Fig. 3. Velocity pulses in active-region fibrils. (a) A downward displacement running from right to left (dark), (b) a similar upward displacement shortly beforehand (bright). Velocity of propagation is 150 $\mathrm{km} \mathrm{s}^{-1}$ in both cases. Successive frames are at 15 -s intervals.

the oscillations occur in individual points, $350-700 \mathrm{~km}$ across, spaced at intervals of about $2300 \mathrm{~km}$.

An important feature of these oscillations is the phase coherency, which extends at times not only over the one plage but over plage regions up to $10^{5} \mathrm{~km}$ apart (Figure 4). The propagation of this phase takes place at velocities of at least $1000 \mathrm{~km} \mathrm{~s}^{-1}$. The duration of coherency of phase is typically $10-20 \mathrm{~min}$, and the total fraction of the time during which phases are coherent has varied from $5-40 \%$. This is an obviously intriguing phenomenon. It may also be of wider importance if further observation confirms Tanaka's suggestion that flares are associated with periods of phase coherence. 

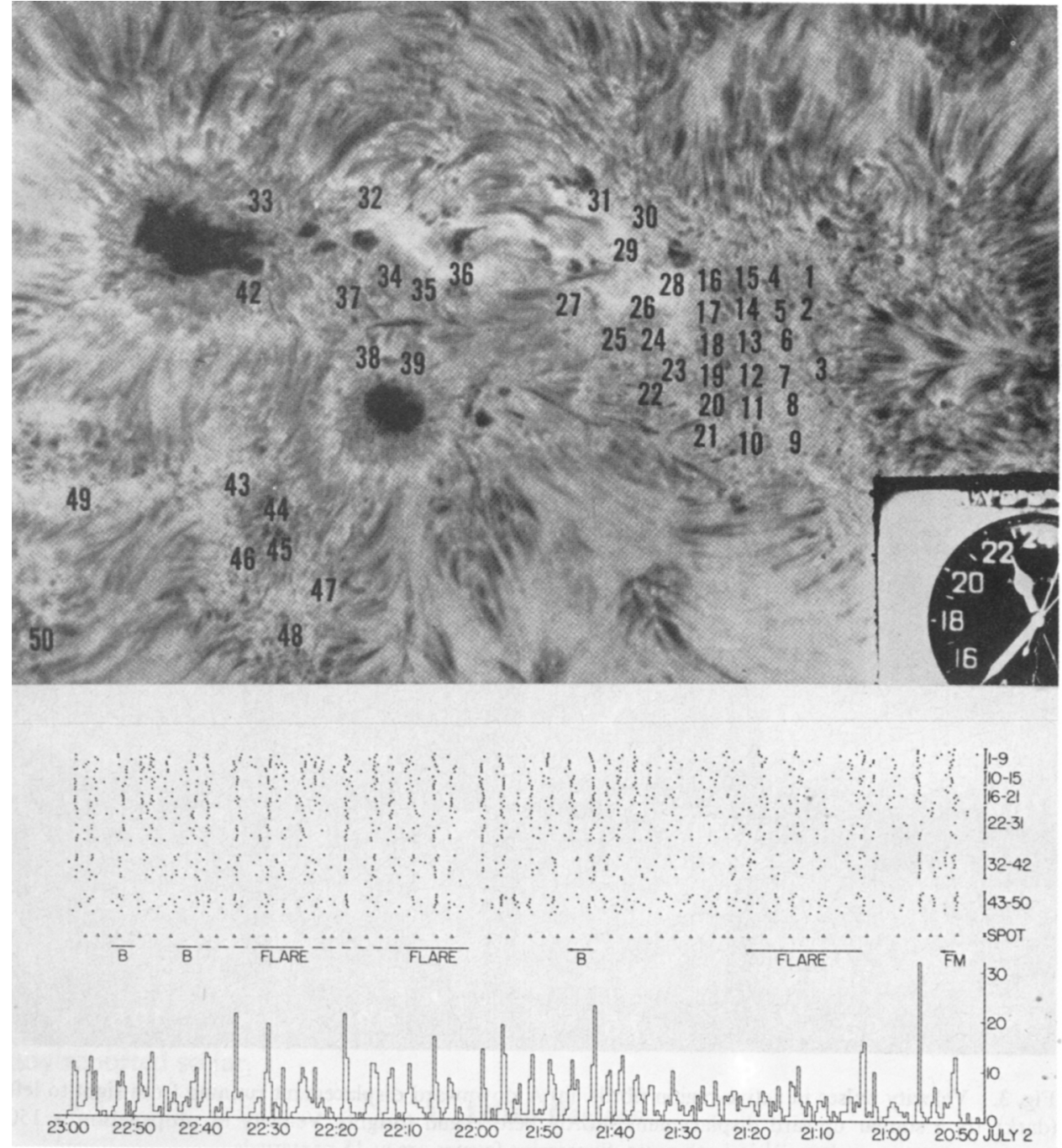

Fig. 4. Top: Active region photographed at $\mathrm{H} \alpha-0.5 \AA$. Middle: Times of maximum intensity at $\mathrm{H} \alpha$ $-0.5 \AA$ (maximum velocity downwards) at individual points in three plages $(1-31,32-42,43-50)$ in above region. Bottom: Histogram showing for all these plages the number of points with simultaneous peaks in oscillation.

Times of flare activity, filament motion (FM) and filament brightening $(B)$ are also shown (Tanaka, private communication).

\section{Concluding Remarks}

We now have direct evidence of Alfvén waves in penumbras and elsewhere in the chromosphere. Their significance in affecting the growth and decay of chromospheric structures is as yet unknown, but this should be a profitable field of study.

The well-known gravitational-acoustic waves are observed up to the highest levels 
accessible to ground-based optical observation, and it is highly desirable that methods be developed now for studying their propagation into the chromosphere-corona transition zone.

Observers have grand opportunities in both these areas, particularly if two-dimensional observations can be made in lines from different levels simultaneously.

\section{References}

Beckers, J. M. and Schultz, R. B.: 1972, Solar Phys. 27, 61.

Beckers, J. M. and Tallant, P. E.: 1969, Solar Phys. 7, 351.

Bhatnagar, A., Livingston, W. C., and Harvey, J. W.: 1972, Solar Phys. 27, 80.

Bhatnagar, A. and Tanaka, K.: 1972, Solar Phys. $24,87$.

Deubner, F.-L.: 1971, Solar Phys. 17, 6.

Elliott, I.: 1969, Solar Phys. 6, 28.

Giovanelli, R. G.: 1972, Solar Phys. 27, 71.

Grossman-Doerth, U. and von Uexküll, Marina: 1971, Solar Phys. $20,31$.

Grossman-Doerth, U. and von Uexküll, Marina: 1973, Solar Phys. 28, 319.

Jensen, E. and Orrall, F. Q.: 1963, Astrophys. J. 138, 252.

Leighton, R. B., Noyes, R. W., and Simon, G. W.: 1962, Astrophys. J. 135, 474.

Liu, S. Y. and Sheeley, N. R.: 1971, Solar Phys. 20, 282.

Orrall, F. Q.: 1966, Astrophys. J. 143, 917.

Sheeley, N. R. and Bhatnagar, A.: 1971, Solar Phys. 18, 195.

Stein, A. and Zirin, H.: 1972, Bull. Amer. Astron. Soc. 4, 392.

Tanenbaum, A. S., Wilcox, J. M., Frazier, E. N., and Howard, R.: 1969, Solar Phys. 9, 328.

\section{DISCUSSION}

Thomas: If there is a $1 \mathrm{~km} \mathrm{~s}^{-1}$ upward velocity corresponding to the outward propagation, why isn't there normal acoustic heating above that region? This is a bigger amplitude than one talks about in the old Biermann-Schwarzschild oscillation business.

Zirin: That's probably what causes the center-line brightening when we do see it.

Giovanelli: Zirin's results and mine are in conflict on this point. I've looked for brightening of the line center and I fail to see it.

Meyer: I was wondering to what Dr Thomas' question referred - to the umbral part or to the penumbral part. In the penumbral part Dr Giovanelli's suggestion was that this was an Alfven wave which is a transverse wave along the magnetic field. This is practically a divergence free wave and would show no compression. It is quite a different wave mode than the ordinary compressional mode and would not give rise to the heating you asked about.

Zirin: I don't think that the divergence in observations between what we observe and what Giovanelli observes is real. First so far as the center line observations are concerned I will show tomorrow a film of plages which also has penumbral waves on it. There is one case where the waves can be seen clearly offband and cannot be seen at all on-band, and we had good contrast. There are other cases where you see them on-band and not off-band. I think there are real differences from spot to spot and that there are cases where they are sometimes better seen as a velocity shift. In velocity they are much more visible than in brightness. I don't understand why they are sometimes seen better off-band than on-band except that it may just indicate where the lines of force are going. Second, our impression that the umbral flashes were exploding and producing an outward wave every second time has been based on observations made at the limit of resolution and should be taken with caution. This is simply an impression that we gathered from looking at films. Certainly the umbral flashes are more frequent than the penumbral waves. I think it is close to two to one but I would not argue strongly that every other one produces a wave. Finally I would add that we have not been able to detect any penumbral waves once the spot is more than four or five days from the centre of the disk. So I think basically we agree on most of the observations and the rest of it is just trying to understand these very subtle phenomena. I think we are just beginning to scratch the surface of what will turn out to be a much more interesting and involved phenomenon. 
Beckers: My slide (see the figure in this discussion) shows observations taken with our universal filter in the $\mathrm{H} \alpha$ line. The time interval is too large to really show the motion of the waves but one can see the waves quite well. For example, in the second set of pictures one can follow the wave through the line centre clearly into the violet wing $(\mathrm{H} \alpha-0.3 \AA)$ but it can hardly be seen in the red wing $(\mathrm{H} \alpha+0.3 \AA)$. The waves are very well visible in the blue wing, marginally visible in line centre and not visible in the red wing. I think

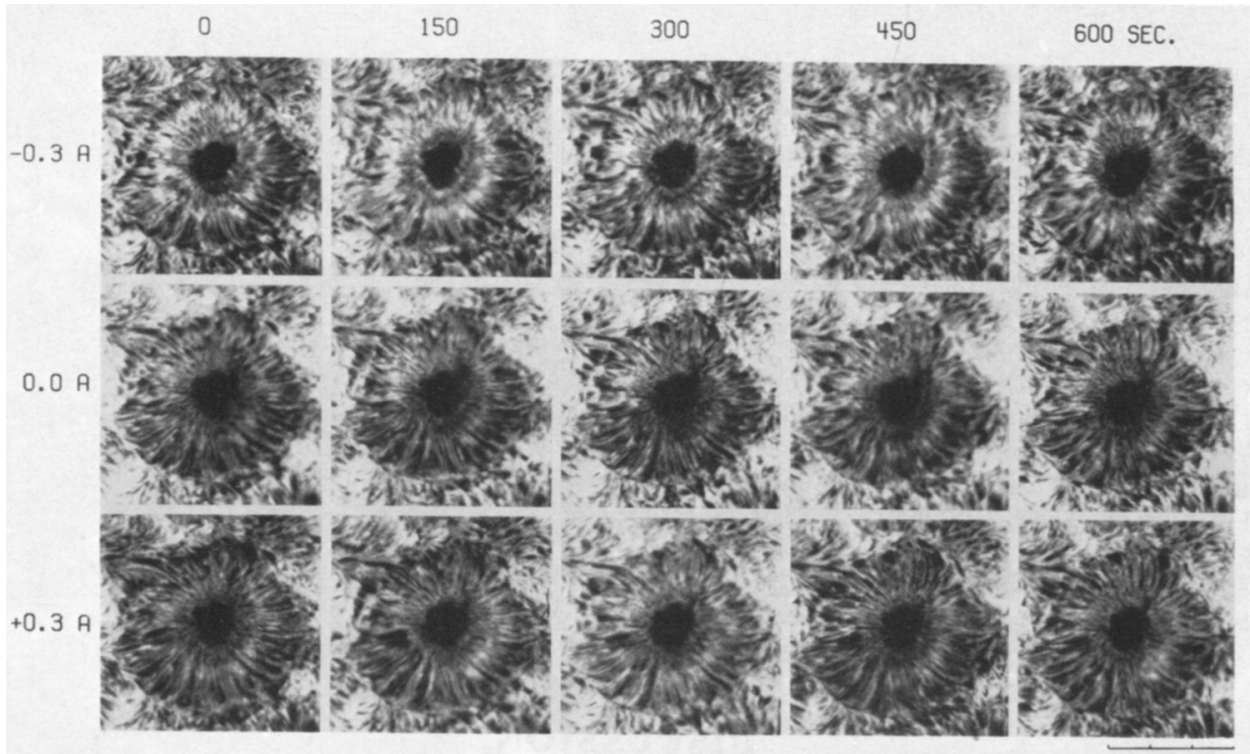

30 ARC SEC.

one sees a relationship between the upward motions and the brightening in the line centre. In other lines or further into the wings of $\mathrm{H} \alpha$ the wave becomes invisible. Waves cannot be seen in the sodium D-lines or the magnesium b-lines. They are best studied in the core of $\mathrm{H} \alpha$.

Wilson: With regard to the invisibility of these waves in lines that lie deeper, as you go down deeper you expect a smaller transverse velocity because of the increased density. Would you expect to see these waves at deeper layers?

Giovanelli: I don't know what the propagation characteristics will be. The magnetic field may be more or less uniform but the densities will vary considerably and complicated refraction effects can be expected. On the other hand the wavelength is an appreciable fraction of the width of the penumbra. The waves disappear before the outer edge of the penumbra.

Sturrock: It is my opinion that any interpretation of waves in terms of an infinite, uniform medium is suspect because in this problem the scale height is small compared to the wavelength. Furthermore, when you compare the Alfvén velocity with the observed wave velocity by inferring a density from the velocity and field strength, you could probably get whatever you need by choosing the right layer. You must take account of the fact that this is a very highly stratified medium and you need to study the modes that exist in a stratified medium not in an infinite homogeneous medium. In this context I wonder whether your statement that the wave is transverse really rules out the acoustic wave. The same can be said about AthayMoreton waves which are produced by flares and propagate on the surface of the Sun. One observes vertical displacements propagating horizontally with high velocities, but Dr Meyer showed that this could be interpreted as the response of the chromosphere to a shock wave travelling in the corona. It is possible that the waves you observe are a similar phenomenon. To sum up I think you must really take into account the stratified nature of the atmosphere and investigate the possible kinds of wave modes that can exist under these circumstances.

Uchida: I would think that there is a possibility that the vertical oscillation in the umbral portion is a secondary velocity induced by the horizontal velocity of the Alfvén wave in a cylinder with a node inside of it. The situation in such a case is fairly different from an Alfvén wave in an infinite medium. Each half of 
the cylinder moves towards each other, for example, with compression in the center, and then each half moves towards the outer boundary, and the material is compressed there, with induced vertical velocity each time. If we consider a situation like this, it may not contradict the fact that you do not observe systematic velocities at the limb. The Alfvén wave may thus be able to carry a considerable amount of energy without being detected except for a small induced velocity parallel to the field.

Giovanelli: It might be possible to test for such a possibility by making observation at different intervals from line center and thereby seeing to different optical depths.

Schmidt: I subscribe to the remark by Dr Sturrock but nevertheless I think that in the umbra the energy flux estimates for the vertical flux cannot be too wrong. In answer to the question by Dr Thomas I think that if you look up the model of Ulmschneider these are just the frequencies you need to escape dissipation in the chromosphere and dissipate in the corona.

Zwaan: Do you find the penumbral waves and the umbral flashes in all spots that you have observed near the center of the disk?

Giovanelli: We have observed them in all that we have looked at. Zirin has not seen them in complex spot groups. I have looked in one complex spot group and seen them, but my statistics are not adequate to dispute Zirin's claims that they do not occur in the complex spot groups.

Zwaan: Then there seems to be a difference between the oscillations you see in $\mathrm{H} \alpha$ and the oscillations seen by Beckers in photospheric lines who found photospheric oscillations in a part of one umbra out of five spots observed, I believe.

Beckers: That was just a one time observation and not an observation of a large number of spots. We saw it in the umbra and an indication of an oscillation existing in the penumbra at a very much longer period. But that was only one spot out of six.

Martin: Ramsey and Phillis at the Lockheed Observatory have also been studying the $\mathrm{H} \alpha$ oscillations in sunspots. They find oscillations in essentially all spots. Their results completely agree with Dr Giovanelli's but they do have one additional interesting example of a sunspot which has an umbral oscillation with a period of $145 \mathrm{~s}$ and can be followed through 8 complete cycles. The variation is only $\pm 5 \mathrm{~s}$ from a completely sinusoidal wave.

Zirin: There was a misunderstanding of Zwaan's question. Zwaan asked if umbral flashes were observed in all spots. We observe umbral flashes in the $\mathrm{K}$ line in all spots. It is just the penumbral waves that we do not see in all spots.

Giovanelli: I think we should be careful not to call them umbral flashes - they are umbral oscillations.

Rosenberg: When you look at penumbral waves, it seems that they spread out with a rather wide opening angle. This contradicts your idea of Alfvén waves. They should be channeled. With regard to umbral oscillations, you said the periods were different for different positions within the umbra and this contradicts the remark by Uchida that the waves are possibly a standing oscillation in a tube. When you look at the ATM pictures, you see flux tubes which must be really very hot - it may be proof that the oscillations do heat the matter in the flux tubes and this is maybe an answer to Dr Thomas' question. There is apparently heating because we see these beautiful arches filled with hot material.

Giovanelli: On the first question I don't see any problem there. I regard an ideal sunspot as a monopole sending lines of force out in every direction. If you go on the hypothesis that these are Alfven waves then you expect the Alfvén waves to go out in a more or less circular manner.

Rosenberg: The way you drew the picture is that one part of the umbra knocks against the field lines. I would expect that this would do something to just those field lines which were disturbed. It's not the whole umbra that goes up and down, is it?

Giovanelli: I see wavefronts which swirl around, expand and do all sorts of extraordinary things. This will cause a wave to propagate out, arriving at perhaps slightly different times at the penumbral-umbral border, but I see these umbral waves starting out and spreading over a very large part of the umbra. In some of the films you may have seen penumbral waves running out quite uniformly, but that is by no means the general rule.

Schatten: I don't see how you can calculate the energy flux associated with the waves without knowing the depth at which these waves are occurring. These waves could extend through the entire convective zone. How do you obtain the wave energy?

Giovanelli: The energy flux I gave is simply the energy flux per unit area at right angles to the propagation of the wave front at the depth where the waves are observed.

Athay: Then it is not valid to compare that with the radiation flux crossing the Sun per unit area in the radial direction?

Giovanelli: I think it is a valid comparison to compare the energy flux crossing unit area normal to the 
wave front with the radiation flux crossing unit area normal to the solar surface.

Wilson: But certainly if there is divergence of the magnetic field then you are reducing the amount of energy that you might calculate.

Beckers: Is the density of about $3 \times 10^{16}$ atoms $\mathrm{cm}^{-3}$ for the depth where you observe these waves consistent with sunspot models? This seems rather high for the region where $\mathrm{H} \alpha$ is formed.

Giovanelli: Over the sunspot the temperature is low, the electron density is very low, and there is very low excitation. Under these conditions it is possible that $\mathrm{H} \alpha$ is formed quite deep in the atmosphere.

Wiehr: Do the penumbral waves run outside the outer border of the penumbra as seen in white light?

Giovanelli: No, they do not.

Wiehr: If they would do so it would be an argument against the Alfven waves because the sunspot magnetic field apparently ends at the outer border of the penumbra. I have another comment concerning your estimate of the Alfvén velocity and the velocity of sound. You ruled out the possibility of interpreting these waves as a sound wave, as well as a possible explanation as gravitational waves. I am not at all convinced about your estimates of the sound velocity and the Alfvén velocity. You must deal with a very inhomogeneous material in the penumbra. It isn't known whether the penumbral waves occur in the bright filaments or the dark filaments in the penumbra. There might be a very large difference in both temperature and density between these two components of the penumbra. This could alter your estimates of the velocity of sound and the Alfven velocity by at least one order of magnitude. Even if you take into account the magnetic field we don't know whether the magnetic field is stronger or weaker in the dark components of the penumbra as compared to the bright components.

Giovanelli: I would remind you that the wave is a transverse wave however.

Beckers: This is for an average penumbra and I don't think it is established yet whether the wave goes in the bright regions or the darker regions. Do you still want an answer to your first question as to why these waves do not penetrate outside of the penumbra. I would suggest that it is because the magnetic field just ceases to exist at that point.

Giovanelli: It's a bit of a problem. I can give an explanation but I'm not very happy with it. My explanation is that the magnetic field is oriented upwards and as the waves go upward along the field they reach a point where there is insufficient optical depth in $\mathrm{H} \alpha$ for the phenomenon to be observed. However I can see some rather nasty problems associated with this explanation.

Beckers: Do you see any change in velocity, either slowing down or speeding up as the wavefront approaches the border of the penumbra.

Giovanelli: I have tried to measure this but have not been able to convince myself that there is any acceleration. My visual impression from watching the films is that there is an acceleration but since I cannot measure it my answer has to be that there is no measurable acceleration.

Zirin: We have not been able to measure any acceleration in the waves we observe and this is one reason why I decided they must be sound waves. It would seem to me strange that the Alfven velocity should remain constant over such a range although maybe it is. Perhaps this just proves that the ratio of $B^{2} / \varrho$ is constant.

Giovanelli: The accuracy with which one can make this measurement is not good. It is difficult enough to measure the velocity and even more difficult to measure an acceleration, particularly when there is little more than one wavelength covering the penumbra. This is something that should be studied in a very large sunspot.

Schmidt: If the polarization is right and the velocity is locally right for Alfven waves then it is up to us theorists to prove that they are not Alfvén waves if this is what we believe. The basic feature of a wave is its polarization and that is just right for the Alfven wave. Also the local velocity of propagation seems to be right. Of course we could propose an explanation that this is a phase velocity associated with another type of wave but after doing some rough computations I am now convinced that the explanation of these waves as Alfven waves is the correct one.

Meyer: May I ask you again. Do these waves end at the exact outer border of the penumbra or do they extend out into the border of the $\mathrm{H} \alpha$ penumbra in the fibrils that extend beyond the white light penumbra?

Giovanelli: The waves end at or before the edge of the penumbra as seen in the white light continuum.

Meyer: I would like to suggest that two of your observations already discussed might point to a somewhat different mode of propagation for your penumbral waves. Dr Wiehr has already commented on the observation that you see these waves in $\mathrm{H} \alpha$ only to a distance coincident with the white light boundary of the penumbra, and Dr Beckers pointed to a rather high value $\left(10^{16} \mathrm{~cm}^{-3}\right.$ for $B=1500 \mathrm{G}$ and a propagation speed of $15 \mathrm{~km} \mathrm{~s}^{-1}$ ) for the inferred density in the level of propagation. Dr Sturrock mentioned the 
problem of propagation in a medium of varying density. But it seems to me that all this loosely suggests the following picture. We know that quite a sizeable amount of magnetic flux leaves the penumbra and from this one has to infer that besides very horizontal penumbral field lines one must have there a large amount of field lines with strong vertical components. If this field configuration is pushed from a pressure disturbance in the umbra a compressional wave should radially travel outwards in which the more vertical field lines are horizontally compressed. Though the amplitude in the more photospheric penumbral level of $10^{16.7} \mathrm{~cm}^{-3}$ density might be rather small, still material would be vertically squeezed upwards along the field lines and in the decreasing density with height might obtain vertical velocities that make it observable in $H \alpha$. Still the main mass involved would be sitting in lower levels and provide the inertia indicated by the $15 \mathrm{~km} \mathrm{~s}^{-1}$ propagation speed. At the outer penumbral boundary one has run out of more vertical field and this might account for the end of the $\mathrm{H} \alpha$ wave pattern. Refraction of cóurse will play a major role for such disturbances, and other difficulties might arise. But it is interesting enough to deserve a model analysis.

Athay: The last series of pictures you showed illustrated a type of motion that appears very similar to what the Sacramento Peak observers have called flagellant motion. Do you think this really is the same phenomena or is there something different?

Giovanelli: I believe the flagellant motions are primarily just the result of Doppler shifts, so the answer to your question is yes! I believe these are the same type of phenomena.

Wilson: Do you have any evidence as to the velocity amplitudes in these waves.

Giovanelli: I would be surprised if it were very large. The estimates given by other people of $1-1 \frac{1}{2}$ $\mathrm{km} \mathrm{s}^{-1}$ might easily be about right.

Newkirk: I am uncertain about your identification of these motions with Alfvén waves. Are the velocities right for this type of wave?

Giovanelli: The velocities that are observed are consistent with Alfvén velocities as nearly as I can determine them. I choose fields and densities that seem to be reasonable and I get the right orders of magnitude for the observed velocities.

Schmidt: I think that the motions in the umbra are probably not Alfvén waves because they seem to be longitudinal. Here we do not have a well-defined field and we cannot make the checks that you have made in the penumbra. In the penumbra I think you have some good arguments that the penumbral waves are Alfvén waves. I say that because we are dealing with a phenomenon which is controlled from below. You cannot control the penumbra from the corona so the arguments applied to the Moreton waves do not apply here.

Giovanelli: I concede immediately that there is a fascinating problem here and that we have a nonuniform magnetic field surrounded by a plasma. I hope we can interest some of the theoreticians in this particular problem.

Deubner: There is another argument against the interpretation of these waves as Alfvén waves. You stated that the velocity in the fibrils was probably 80 to $100 \mathrm{~km} \mathrm{~s}^{-1}$ and that close to the spot where the field is much stronger the velocity is of the order of $12 \mathrm{~km} \mathrm{~s}^{-1}$ and I think this is a contradiction.

Giovanelli: When I use existing chromospheric models and sunspot models the models differ enough that I still get the right order of magnitudes for velocities. 